\title{
ARTICLE
}

\section{Fluctuations in the electron system of a superconductor exposed to a photon flux}

\author{
P.J. de Visser ${ }^{1,2}$, J.J.A Baselmans ${ }^{1}$, J. Bueno ${ }^{1}$, N. Llombart $^{3}$ \& T.M. Klapwijk ${ }^{2}$
}

In a superconductor, in which electrons are paired, the density of unpaired electrons should become zero when approaching zero temperature. Therefore, radiation detectors based on breaking of pairs promise supreme sensitivity, which we demonstrate using an aluminium superconducting microwave resonator. Here we show that the resonator also enables the study of the response of the electron system of the superconductor to pair-breaking photons, microwave photons and varying temperatures. A large range in radiation power (at $1.54 \mathrm{THz}$ ) can be chosen by carefully filtering the radiation from a blackbody source. We identify two regimes. At high radiation power, fluctuations in the electron system caused by the random arrival rate of the photons are resolved, giving a straightforward measure of the optical efficiency $(48 \pm 8 \%)$ and showing an unprecedented detector sensitivity. At low radiation power, fluctuations are dominated by excess quasiparticles, the number of which is measured through their recombination lifetime.

\footnotetext{
${ }^{1}$ SRON Netherlands Institute for Space Research, Sorbonnelaan 2, 3584 CA, Utrecht, The Netherlands. ${ }^{2}$ Kavli Institute of NanoScience, Faculty of Applied Sciences, Delft University of Technology, Lorentzweg 1, 2628 CJ, Delft, The Netherlands. ${ }^{3}$ Terahertz Sensing Group, Faculty of Electrical Engineering, Mathematics and Computer Science, Delft University of Technology, Mekelweg 4, 2628 CD, Delft, The Netherlands. Correspondence and requests for materials should be addressed to P.J.de.V. (email: p.j.devisser@tudelft.nl) or to J.J.A.B. (email: j.baselmans@sron.nl).
} 
n a superconductor well below its critical temperature, the majority of the electrons is bound in a condensate of Cooper pairs. The further the superconductor is cooled down, the closer it gets to its ground state, where all the quasiparticles are condensed to pairs. Due to the low gap energy, the superconductor is sensitive to disturbances from the environment to which it couples. In most experiments, this sensitivity is undesirable, but it is particularly suited for detection of radiation. The superconductor can interact with its environment due to either photons or phonons. Photons with an energy higher than the energy gap break up Cooper pairs into quasiparticles. The change in the number of quasiparticles and Cooper pairs changes the electrodynamic response of the superconductor, which can be measured using a microwave resonator ${ }^{1}$. Quasiparticles give rise to microwave losses and the Cooper pairs to a kinetic inductance ${ }^{2}$. In steady state, the number of quasiparticles fluctuates in time around a constant average value. A measurement of the spectrum of these fluctuations allows for a characterization of the quasiparticle system when exposed to pairbreaking photons, microwave photons or to changes in the bath temperature. The characteristic timescale of the fluctuations, the quasiparticle recombination time, is inversely proportional to the number of quasiparticles ${ }^{3}$, and is therefore a measure of this number. These fluctuation phenomena are a monitor of the superconducting state and reveal the physical mechanisms that are at the heart of pair breaking in a superconductor.

We study these processes in a superconducting pair-breaking detector formed by a $50 \mathrm{~nm}$ thick Al film. The ideal pair-breaking detector can either count single photons while its sensitivity is limited by Fano noise, or is photon integrating and limited by photon noise, the noise from the photon source itself ${ }^{4}$. In both cases, a high optical efficiency is required. The principle of radiation detection due to pair breaking with superconducting microwave resonators was proposed in 2003 (refs 1,5). Since then, the most important source of excess noise ${ }^{1}$, due to dielectric two level systems, has been thoroughly studied ${ }^{6-9}$. Here we report on an all-aluminium antenna-coupled microwave resonator detector (Fig. 1a) that is limited only by fluctuations in the electron system that are fundamentally connected to the physical process of pair breaking.

\section{Results}

Detector sensitivity. We use a blackbody with a variable temperature $(3-25 \mathrm{~K})$ and eight optical filters, which define an optical band around $1.54 \mathrm{THz}$, as shown schematically in Fig. 1b. The radiation power can be changed from $3 \times 10^{-21} \mathrm{~W}$ to $7 \times 10^{-13}$ W. At powers ranging from $0.1-700 \mathrm{fW}$, the sensitivity is only limited by the random arrival rate of the photons, which is evident through the measured power dependence of the noise equivalent power (NEP) as shown in Fig. 1c. At lower radiation powers, we observe a power-independent NEP. This is consistent with generation-recombination noise due to the presence of excess quasiparticles ${ }^{10,11}$. Excess quasiparticles are a general concern for superconducting devices ${ }^{12-18}$. In this case, they are generated by the microwave readout power ${ }^{19,20}$. As shown in Fig. 1c, they impose a lower limit to the NEP of this detector of $3.8 \pm 0.6 \times 10^{-19} \mathrm{WHz}^{-1 / 2}$, which is the lowest reported so far for this type of detectors.

Design of the experiment. The detector is based on a lens antenna-coupled superconducting microwave resonator. The resonator is an open ended, half wave, coplanar waveguide resonator, where the central strip (with a width of $3 \mu \mathrm{m}$ ) is isolated from the ground plane. The resonators all have different lengths and therefore different resonant frequencies, enabling the readout of all resonators using a single coaxial line. Radiation is focused by a silicon lens onto an X-slot antenna ${ }^{21}$, optimized for

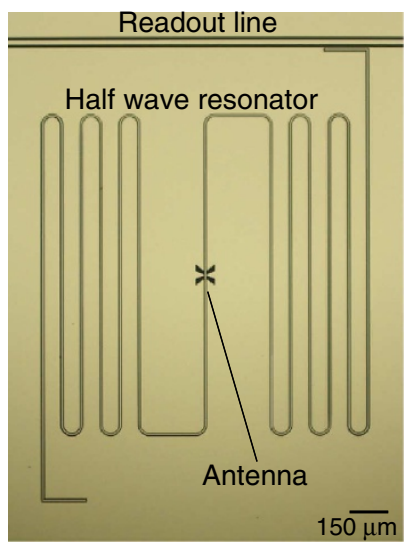

b

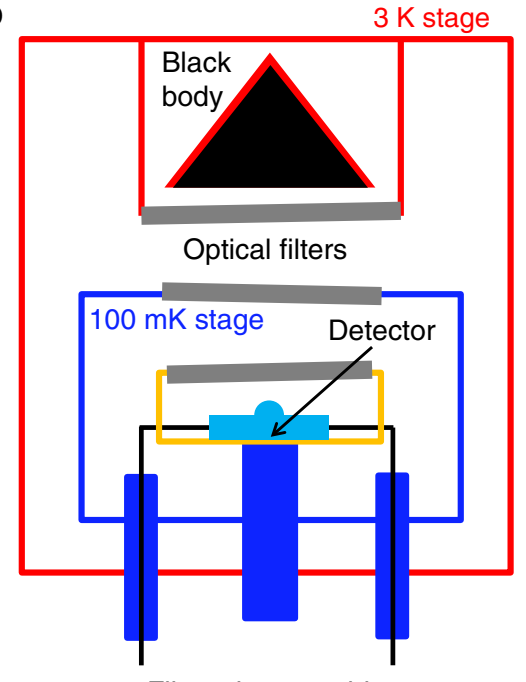

C

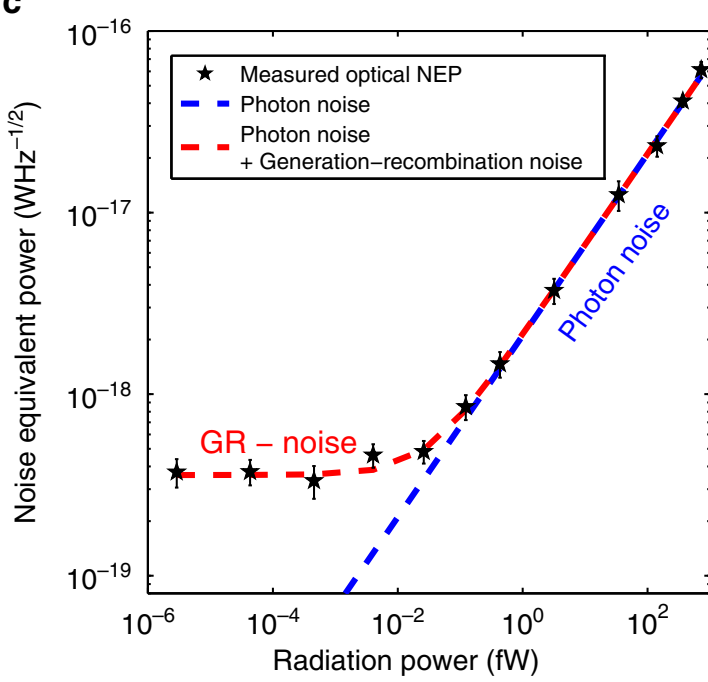

Figure 1 | Overview of the experiment and main results. (a) A picture of one antenna-coupled microwave resonator. It consists of a half wavelength coplanar waveguide microwave resonator with two open ends, capacitively coupled to a microwave readout line. In the middle, it has an X-slot antenna to receive terahertz radiation. The ground plane and central strip of the resonator are respectively 100 and $50 \mathrm{~nm}$ thick layers of aluminium. (b) Schematic of the setup for measurements at various radiation powers. A blackbody with a variable temperature illuminates the lens antenna-coupled resonators through three stacks of filters, which define a passband around $1.54 \mathrm{THz}$. Because of the box-in-box configuration at $100 \mathrm{mK}$ and the coax cable filters, the device is well shielded from stray light. (c) The optical sensitivity is expressed in NEP as a function of radiation power at a frequency of $20 \mathrm{~Hz}$. We observe two regimes: above $0.1 \mathrm{fW}$ the NEP increases with $\sqrt{P_{\text {rad }}}$, indicative of photon noise, whereas below $0.1 \mathrm{fW}$ the NEP saturates. The blue line is the photon noise limit as a function of power, with the optical efficiency (48\%) taken into account. For the red dashed line, the generation-recombination noise limit due to excess quasiparticles is taken into account, based on a quasiparticle recombination time of $3 \mathrm{~ms}$. The error bars $(1 \mathrm{s.d}$.) are combined statistical uncertainties from the noise level and responsivity. 
broad band detection from 1.4-2.8 THz. Radiation coupled to the antenna is launched as a travelling wave into the waveguide ${ }^{21}$, where it is absorbed by breaking Cooper pairs (the gap energy $\Delta=188 \mu \mathrm{eV}$ ). The created quasiparticles, which can diffuse over several millimetres before they recombine, are confined to the central strip. The central strip layer is $50 \mathrm{~nm}$ thick, and the ground plane layer $100 \mathrm{~nm}$. The thin central strip layer gives higher response and ensures that most of the radiation is absorbed in that central strip, due to its higher resistance (see Methods). The thick ground plane reduces antenna losses. An advantage of the geometry, shown in Fig. 1a, is that it can also be used at other radiation frequencies by only changing the antenna.

The sample is cooled in a pulse tube precooled adiabatic demagnetization refrigerator. The sample stage is carefully shielded from stray light from the $3 \mathrm{~K}$ stage of the cooler, using a box-in-a-box concept with optical filters at each stage, as well as coax cable filters in the outer box ${ }^{22}$. The photon source is a blackbody with a variable temperature between 3 and $25 \mathrm{~K}$ (Methods). The system is schematically depicted in Fig. 1b. Eight optical filters in series define an optical band-pass of $0.1 \mathrm{THz}$ centred around $1.54 \mathrm{THz}$. Three filter stacks are essential to eliminate filter heating. The filter transmission of the three filter stages is shown in Fig. 2a. The curves of spectral radiance for high and low blackbody temperature indicate a large tuning range in radiation power $\left(P_{\text {rad }}\right)$. In fact, $P_{\text {rad }}$ can be varied between $3 \mathrm{zW}$ $\left(1 \mathrm{zW}=10^{-21} \mathrm{~W}\right)$ and $1 \mathrm{pW}$ (Supplementary Fig. S1). Practically, this experiment allows us to switch from a regime where the number of quasiparticles is fully determined by the radiation to a regime with a negligible number of optically created quasiparticles. We put a polarizing wire grid just before the detector to make sure the detector only receives radiation in the polarization direction of the antenna.

Operation principle. The number of quasiparticles is measured through a measurement of the complex conductivity of the superconductor. The real part of the conductivity, $\sigma_{1}$, is due to the quasiparticles and resistive. The imaginary part, $\sigma_{2}$, is due to the kinetic inductance of the Cooper-pair condensate ${ }^{2}$. When the radiation power or the bath temperature is increased, more quasiparticles are generated, which changes both $\sigma_{1}$ and $\sigma_{2}$. The kinetic inductance increases, which leads to a lower resonant frequency $f_{0}=1 / 2 l \sqrt{\left(L_{\mathrm{g}}+L_{\mathrm{k}}\right) C}$, where $l$ is the length of the resonator, $L_{\mathrm{g}}$ the geometrical inductance, $L_{\mathrm{k}}$ the kinetic inductance and $C$ the capacitance of the line, all per unit length. The losses at microwave frequencies also increase, leading to a shallower resonance curve. Measurements of the resonance curves for various radiation powers are shown in Fig. 2b. In a practical detection scheme, one typically uses an amplitude, $A$, and a phase, $\theta$, referred to the resonance circle in the complex plane ${ }^{6}$, as shown in Fig. 2c. The amplitude response originates from a change in resistance, whereas the phase changes due to the kinetic inductance. We have only used the amplitude response in this experiment, because the phase noise is too high for this resonator (Supplementary Note 1).

The NEP is a convenient quantity to compare the spectra of quasiparticle fluctuations in different regimes, as shown in Fig. 1c. The NEP of the resonator amplitude is experimentally determined from a measurement of the noise spectrum $\left(S_{A}\right)$ and the responsivity to radiation $\left(d A / d P_{\mathrm{rad}}\right)$ and given by

$$
\operatorname{NEP}(f)=\sqrt{S_{A}(f)}\left(\frac{d A}{d P_{\text {rad }}}\right)^{-1} \sqrt{1+\left(2 \pi f \tau_{\mathrm{qp}}\right)^{2}}
$$

with $P_{\text {rad }}$ the radiation power and $f$ the modulation frequency. $d A / d P_{\mathrm{rad}}$ is obtained experimentally by a linear fit to a measurement of $A$ where $P_{\text {rad }}$ is slowly varied around the power of interest (see Methods). The measured microwave response upon a change in $P_{\text {rad }}$ is shown in Fig. 2c. The last factor in equation (1) arises because the quasiparticle system cannot respond to fluctuations that are faster than the quasiparticle recombination time, $\tau_{\mathrm{qp}}$.

Photon-induced quasiparticle fluctuations. If the average number of quasiparticles is dominated by the absorbed optical photons, the number of quasiparticles fluctuates in time due to two contributions. One is fundamental to every power-integrating detector and due to the random arrival rate of the photons, which induces a random generation of quasiparticles. The power spectral density of fluctuations in the resonator amplitude due to this
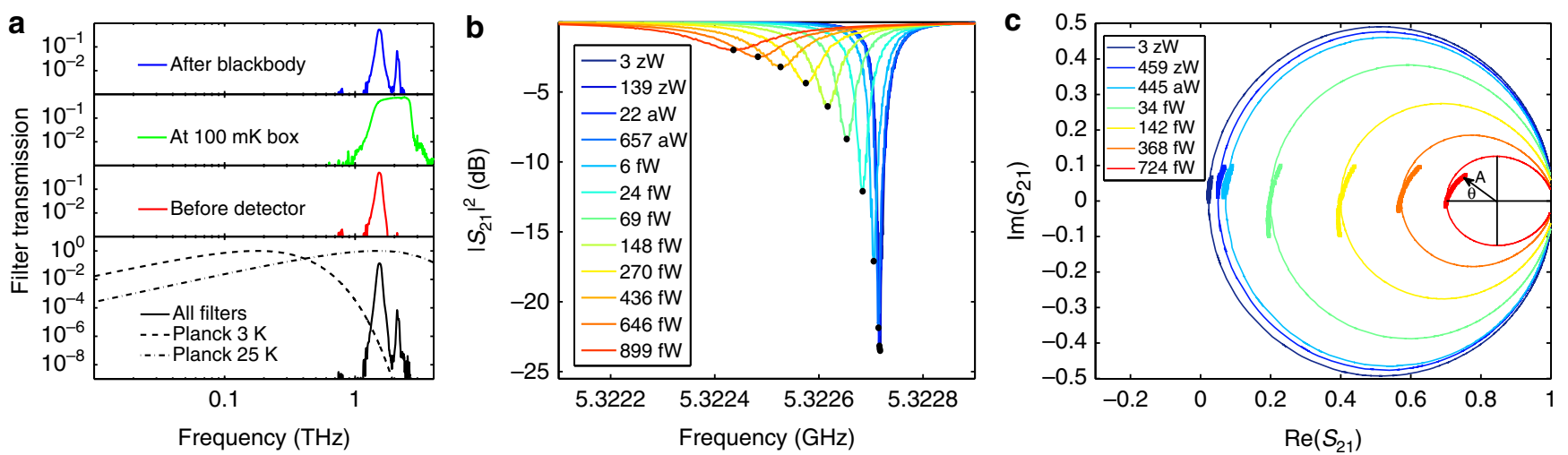

Figure 2 I Response to radiation. (a) Filter transmission characteristics of the three stacks of optical filters in the setup (Fig. 1b). The first and third set of filters have a low-pass, a band-pass and a high-pass filter. The second set (at the $100 \mathrm{mK}$ box) has only a high- and a low-pass filter. In the bottom panel the total transmission of these eight filters is shown. We also show the normalized spectral radiance (Planck's law) at two blackbody temperatures, which demonstrate the large tunability in radiation power in this spectral range. Note that especially for low blackbody temperatures only a fraction $10^{-6}$ of the total power is in the spectral range of interest. The rejection of the rest of the power requires the eight consecutive filters. (b) The magnitude of the microwave transmission $\left|S_{21}\right|^{2}$, measured as a function of frequency for various radiation powers as shown in the legend. At higher power, more quasiparticles are created, which give a higher resistance and inductance and therefore lead to a lower resonant frequency and a shallower dip. The dots show the resonant frequency at each power. (c) The resonance circle for a selection of radiation powers (legend), measured as a function of frequency (lines). The squares show the response upon a small change in the radiation power measured at constant frequency, the resonant frequency of each circle. In the last circle, we show how that response is translated into an amplitude, $A$, and a phase, $\theta$. 
photon noise is given by ${ }^{4}$

$$
S_{A}^{\mathrm{P}}(f)=2 h F P_{\mathrm{rad}}\left(1+\eta_{\mathrm{opt}} B\right) \frac{\left(d A / d P_{\mathrm{rad}}\right)^{2}}{1+\left(2 \pi f \tau_{\mathrm{qp}}\right)^{2}},
$$

where the first term is the spectrum of the photon (power) fluctuations and the second term describes the resonator response upon a change in the radiation power. $F$ is the frequency of the optical photons and $h$ Planck's constant. The factor $\left(1+\eta_{\text {opt }} B\right)$ is the correction to Poissonian statistics due to photon bunching for a single mode, with $\eta_{\text {opt }}$ the optical efficiency and $B$ the mode occupation $^{23}$. The photon bunching contribution is negligible for the here measured power range (Supplementary Fig. S1c). Equation (2) is valid as long as $\tau_{\mathrm{qp}} \gg \tau_{\text {res }}$, which holds in this experiment since the response time of the resonator, given by $\tau_{\text {res }}=Q / \pi f_{0}$, is $6 \mu$ s. $Q$ is the quality factor of the resonator.

Because of the pair-breaking nature of the radiation absorption, a second noise mechanism arises due to random recombination of the quasiparticles that are generated by the photons. This is half the generation-recombination noise that arises in thermal equilibrium ${ }^{24}$, because generation noise is already contained in equation (2). The spectrum is given by

$$
S_{A}^{\mathrm{R}}(f)=\frac{2 N_{\mathrm{qp}} \tau_{\mathrm{qp}}}{1+\left(2 \pi f \tau_{\mathrm{qp}}\right)^{2}}\left(\frac{d A}{d N_{\mathrm{qp}}}\right)^{2},
$$

with $N_{\mathrm{qp}}$ the number of quasiparticles and $d A / d N_{\mathrm{qp}}$ the responsivity of $A$ to a change in $N_{\mathrm{qp}}$. Quasiparticle number fluctuations can be converted to power fluctuations through $\eta_{\mathrm{pb}} \eta_{\mathrm{opt}} P_{\mathrm{rad}}=N_{\mathrm{qp}} \Delta / \tau_{\mathrm{qp}} . \eta_{\mathrm{opt}}$ is the optical efficiency, the efficiency with which power in front of the lens is absorbed in the detector. $\eta_{\mathrm{pb}} \approx 0.57$ is the pair-breaking efficiency ${ }^{25}$, the efficiency with which absorbed radiation power is converted into quasiparticles. For small changes in the quasiparticle number, $d P_{\mathrm{rad}} / d N_{\mathrm{qp}}=$ $\Delta /\left(\tau_{\mathrm{qp}} \eta_{\mathrm{pb}} \eta_{\mathrm{opt}}\right)$ and therefore $d A / d P_{\mathrm{rad}}=\tau_{\mathrm{qp}} \eta_{\mathrm{pb}} \eta_{\mathrm{opt}} / \Delta \cdot\left(d A / d N_{\mathrm{qp}}\right)$. From equations (2) and (3), the relative contribution of photon noise compared with recombination noise is given by $h F(1+m B) \eta_{\mathrm{pb}} \eta_{\mathrm{opt}} / \Delta=10$ at all $P_{\mathrm{rad}}$, for $F=1.54 \mathrm{THz}$ and $\eta_{\text {opt }}=0.5$.

The NEP due to photon noise and recombination noise (equations (1-3)), for $f<1 /\left(2 \pi \tau_{\mathrm{qp}}\right)$, is given by

$$
\mathrm{NEP}_{\text {photon }}=\sqrt{\frac{2 P_{\mathrm{rad}} h F\left(1+\eta_{\mathrm{opt}} B\right)+2 \Delta P_{\mathrm{rad}} / \eta_{\mathrm{pb}}}{\eta_{\mathrm{opt}}}},
$$

which is shown as the blue dashed line in Fig. 1c. The three contributions in equation (4) are compared in Supplementary Fig. S1c.

In thermal equilibrium $N_{\mathrm{qp}}$ is related to $\tau_{\mathrm{qp}}$ through

$$
N_{\mathrm{qp}}=\frac{1}{\tau_{\mathrm{qp}}} \frac{\tau_{0} N_{0}\left(k_{\mathrm{B}} T_{\mathrm{c}}\right)^{3} V}{2 \Delta^{2}}
$$

where $N_{0}$ is the single spin density of states at the Fermi level, $V$ the volume, $k_{\mathrm{B}}$ Boltzmann's constant, and $\tau_{0}$ the characteristic electron-phonon interaction time $\mathrm{e}^{3}$. We take $N_{0}=1.72 \times 10^{10} \mu \mathrm{m}^{-3} \mathrm{eV}^{-1}$ and $V=0.6 \times 10^{3} \mu \mathrm{m}^{3}$, half the volume of the central strip of the resonator (see Methods). In this experiment equation (5) can be applied under the assumption that the response of the resonator amplitude is dominated by quasiparticle generation and recombination. We have shown earlier in a similar aluminium device that $N_{\mathrm{qp}}$ and $\tau_{\mathrm{qp}}$ are related by equation (5) for thermally generated quasiparticles. This equation is also expected to hold in non-equilibrium conditions due to optical excitations ${ }^{26}$ or due to microwave readout power dissipation at low bath temperatures ${ }^{20}$.

The fluctuations in the resonator amplitude were measured as a function of radiation power at a constant bath temperature of
$120 \mathrm{mK}$ (Methods). The resulting power spectral densities are shown in Fig. 3a for a selection of radiation powers and a constant microwave readout power (the power on the readout line) of $-88 \mathrm{dBm}$. We observe that the spectra show a roll-off, the frequency of which increases as a function of radiation power, due to the decreasing quasiparticle recombination time. The other phenomena in the noise spectrum at higher frequencies (the bump at $20 \mathrm{kHz}$, a second higher frequency roll-off and amplifier noise, see Supplementary Fig. S2) are understood and can be accounted for (see Supplementary Note 2). The quasiparticle recombination times from the roll-off in the spectra are shown as a function of radiation power in Fig. $3 \mathrm{~b} . N_{\mathrm{qp}}$, calculated using equation (5) is shown on the right axis. Since $\eta_{\mathrm{pb}} \eta_{\mathrm{opt}} P_{\mathrm{rad}}=N_{\mathrm{qp}} \Delta /$ $\tau_{\mathrm{qp}}$ and $N_{\mathrm{gp}} \propto 1 / \tau_{\mathrm{qp}}$ (equation (5)), $\tau_{\mathrm{qp}}$ is expected to scale as $\tau_{\mathrm{qp}} \propto P_{\mathrm{rad}}^{-1 / 2 \mathrm{p}}$. A fit to the measured recombination time as a function of $P_{\text {rad }}$ results in $\tau_{\mathrm{qp}} \propto P_{\mathrm{rad}}^{-0.50 \pm 0.03}$, which agrees very well with the expected behaviour. Within the measurement accuracy, the same coefficient is measured for other microwave readout powers. The quasiparticle recombination time saturates below about $0.1 \mathrm{fW}$. This saturation in the recombination time is commonly observed ${ }^{27}$, but not yet understood for all materials. Here the saturation is consistent with the presence of excess quasiparticles, which we have demonstrated in measurements on a very similar $\mathrm{Al}$ resonator ${ }^{11}$.

Phonon-induced quasiparticle fluctuations. Excess quasiparticles give rise to quasiparticle number fluctuations $s^{11,19}$. To verify that the spectra in the saturation regime show these fluctuations, we change the number of quasiparticles by varying the number of phonons (the bath temperature) at the same microwave power. The amplitude spectrum is shown for bath temperatures ranging from $90-255 \mathrm{mK}$ in Fig. $3 \mathrm{c}$. The blackbody temperature is kept at $3.2 \mathrm{~K}$, so there are less than 100 quasiparticles due to the radiation power in the sensitive volume. The amplitude spectrum due to quasiparticle fluctuations can be described as ${ }^{10,11} S_{A}^{\mathrm{GR}}(f)=2 S_{A}^{\mathrm{R}}(f)$, because here both generation and recombination are considered. The noise level, which is proportional to $N_{\mathrm{qp}} \tau_{\mathrm{qp}}$, is expected to be constant as a function of temperature (see equation (5)), which we indeed observe in Fig. $3 c$. We assume here that $d A / d N_{\mathrm{qp}}$ is constant for this temperature range ${ }^{26}$. The quasiparticle recombination time extracted from these spectra is plotted as a function of temperature in Fig. 3d. We observe the same saturation level in the recombination time as in Fig. $3 \mathrm{~b}$ where the radiation power was changed. $\tau_{0}=303 \pm 14 \mathrm{~ns}$ is obtained from a fit to the measured $\tau_{\mathrm{qp}}$ as a function of temperature ${ }^{3,11} \cdot \tau_{0}$ is slightly different from earlier results $\left(458 \mathrm{~ns}^{11}\right)$, which could be due to the higher resistivity and $T_{\mathrm{c}}$ of the $\mathrm{Al}^{3}$.

We have now verified that the noise spectra in the regime of low radiation power (below $0.1 \mathrm{fW}$ ) are consistent with quasiparticle number fluctuations. The optical NEP due to quasiparticle number fluctuations is given by

$$
\mathrm{NEP}_{\mathrm{GR}}=\frac{2 \Delta}{\eta_{\mathrm{pb}} \eta_{\mathrm{opt}}} \sqrt{\frac{N_{\mathrm{qp}}}{\tau_{\mathrm{qp}}}} .
$$

Using this equation, the saturation of the NEP in Fig. 1c is consistent with the measured saturation in the quasiparticle recombination time (Fig. 3b) and the number of quasiparticles inferred from that measurement.

If we return to the photon-induced fluctuations in Fig 3a, we observe that the noise level becomes constant at the highest radiation powers as well. This constant level is expected when equations (2) and (3) are rewritten in terms of $N_{\mathrm{qp}} \tau_{\mathrm{qp}}$. The noise level is higher than in Fig. 3c, because there is both photon noise and recombination noise. 

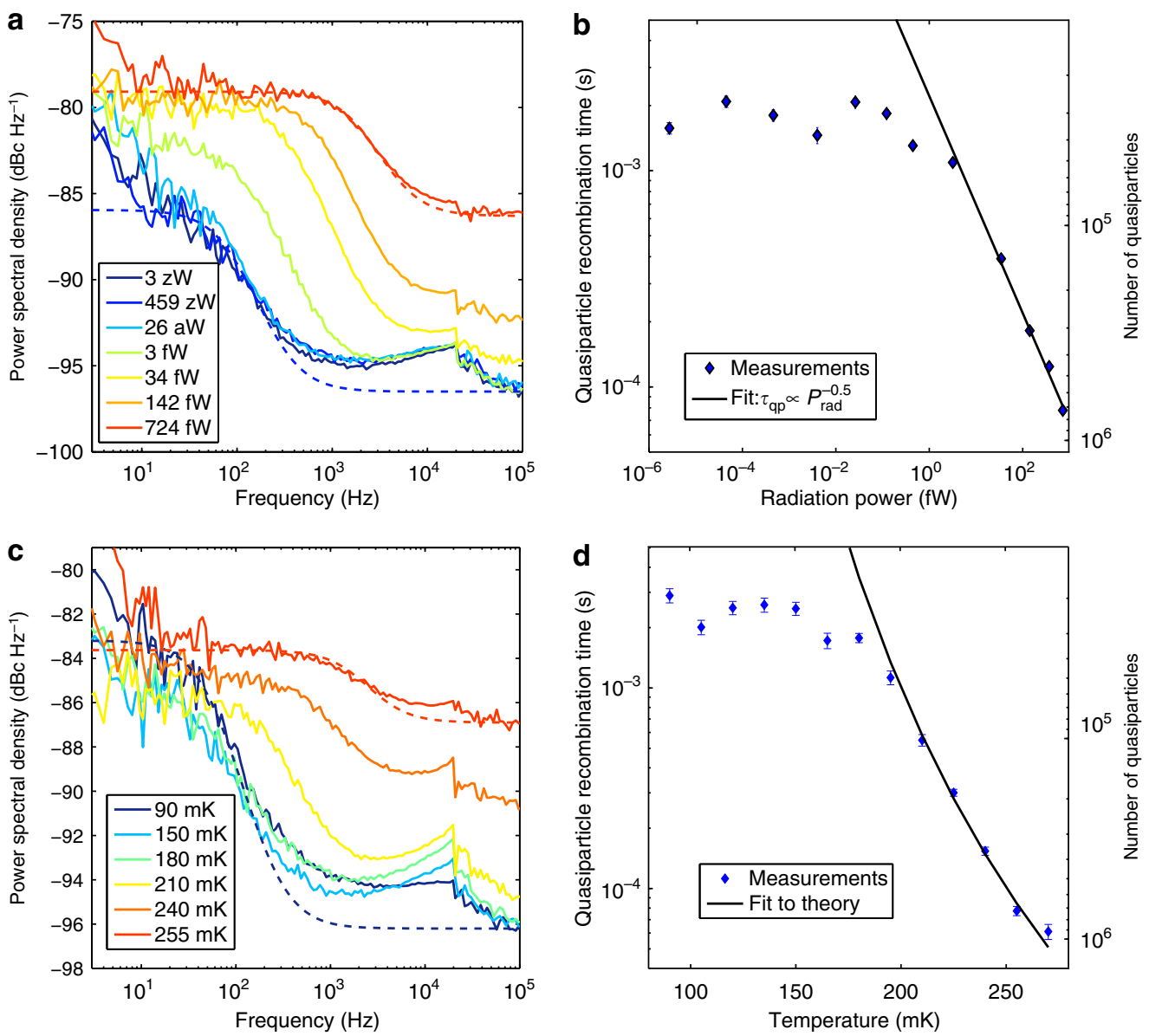

Figure 3 | Quasiparticle fluctuations. (a) Power spectral density of the resonator amplitude as a function of frequency for different radiation powers at a constant bath temperature of $120 \mathrm{mK}$ and a constant microwave readout power of $-88 \mathrm{dBm}$. Lorentzian fits to the spectra at the lowest and highest temperatures (dashed lines) show how the quasiparticle recombination time can be extracted from the spectra. A noise floor due to amplifier noise is added to the fitted roll-off. (b) The quasiparticle recombination time as a function of radiation power obtained from the roll-off frequency in the measured spectra. The error bars denote statistical uncertainties from the fitting procedure $(1 \mathrm{s.d}$.). The line is a power law fit to the last five points (where $\tau_{\mathrm{qp}}$ does not saturate): $\tau_{\mathrm{qp}} \propto P_{\text {rad }}^{-0.50 \pm 0.03}$. The right axis shows the number of quasiparticles corresponding to the measured recombination time. (c) Power spectral density of the resonator amplitude as a function of frequency for different bath temperatures at a microwave readout power of $-88 \mathrm{dBm}$. As expected, the level of the spectrum stays constant and the roll-off frequency increases with increasing temperature, corresponding to a decreasing recombination time. At the highest two temperatures, the spectral level starts to rise, because the amplifier noise starts to dominate.

(d) Quasiparticle recombination time as a function of temperature as extracted from the spectra. The error bars are $1 \mathrm{~s}$.d. The solid line is the theoretical expectation for the recombination time from ref. 3. The right axis shows the number of quasiparticles corresponding to the measured recombination time.

Noise equivalent power. The measured NEP, obtained by using equation (1) together with the measured $S_{A}, d A / d P_{\text {rad }}$ and $\tau_{\mathrm{qp}}$, is shown for various radiation powers in Fig. 4. The NEP measurement was done at a range of microwave readout powers. The results shown in Figs $1 \mathrm{c}$ and 4 are at the readout power with the minimum NEP for that radiation power. The measured $d A$ / $d P_{\text {rad }}$ and $S_{A}$ that constitute the NEP are shown in the Supplementary Fig. S3. Note that the levels of these $S_{A}$ are different from those in Fig. 3a because of the strong dependence of $d A / d P_{\text {rad }}$ on the microwave readout power.

The measured optical NEP at $20 \mathrm{~Hz}$ is shown as a function of radiation power in Fig. $1 \mathrm{c}$ as our main result. At radiation powers of $0.1 \mathrm{fW}$ and higher, the NEP scales with $\sqrt{P_{\text {rad }}}$, as expected from the photon noise limit given by equation (4). In this regime, the optical efficiency is obtained by fitting equation (4) to the measured NEP. The result is shown as the blue line in Fig. 1c, which gives $\eta_{\mathrm{opt}}=0.48 \pm 0.08$ for a single polarization, consistent with electromagnetic simulations of the antenna (Methods).

Below $0.1 \mathrm{fW}$, the NEP saturates at $3.8 \pm 0.6 \times 10^{-19} \mathrm{WHz}^{-1 / 2}$. We have seen that generation-recombination noise due to excess quasiparticles dominates the noise spectra in this regime. From the measured recombination time ( $3 \mathrm{~ms}$, see Fig. $5 \mathrm{~b}$ ), we calculate $N_{\mathrm{qp}}$ using equation (5). The sum of equations (4) and (6) is shown as the red dashed line in Fig. 1c and gives a good account of the measured NEP. The limit of $3.8 \times 10^{-19} \mathrm{WHz}^{-1 / 2}$ is in good agreement with predictions based on dark experiments ${ }^{19}$.

Excess quasiparticles due to the readout power. From recent dark experiments ${ }^{19}$ (without blackbody source) and simulation ${ }^{20}$, we expect that the excess quasiparticles at low radiation power are due to microwave readout power dissipation. In an optical experiment, excess quasiparticles should lead to a decrease in the optical response. The measured responsivity is shown in Fig. $5 \mathrm{a}$ as a function of $P_{\text {read. }}$. Indeed, the responsivity decreases with increasing $P_{\text {read }}$, consistent with excess quasiparticles due to microwave absorption. A power law fit to the responsivity versus readout power results in $d A / P_{\text {rad }} \propto P_{\text {read }}^{-0.4 \pm 0.1}$. Figure $5 \mathrm{~b}$ shows $\tau_{\mathrm{qp}}$, extracted from the noise spectra, as a function of $P_{\text {read. }} . \tau_{\mathrm{qp}}$ increases when $P_{\text {read }}$ decreases, which is also consistent with quasiparticle generation by the micro- 


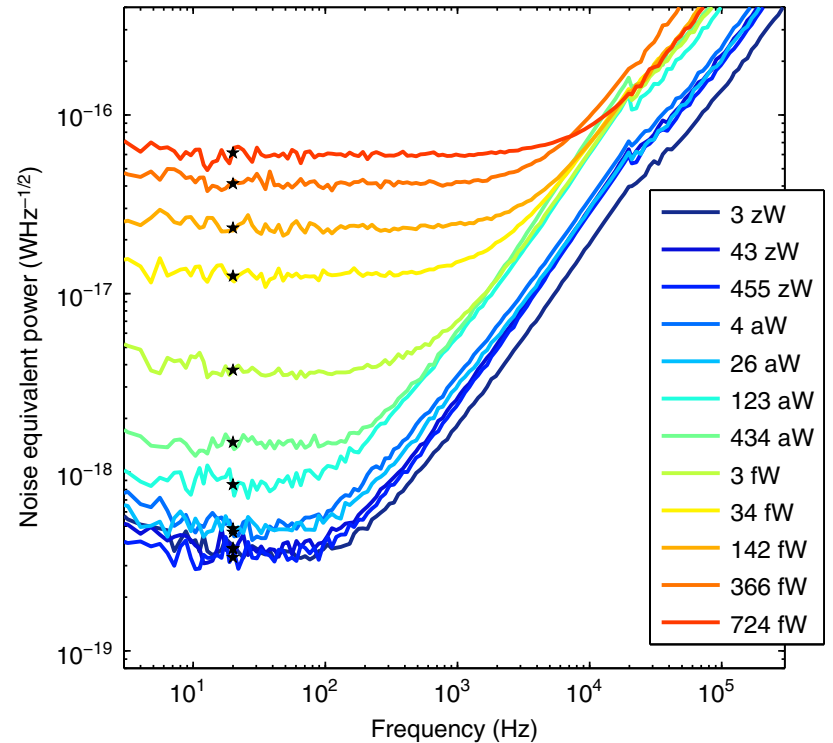

Figure 4 | Noise equivalent power. Measured optical NEP in the resonator amplitude as a function of frequency for different radiation powers. The corner frequency of each spectrum corresponds to the quasiparticle recombination time as plotted in Fig. 3b. The stars indicate the NEP at the reference frequency of $20 \mathrm{~Hz}$, which is chosen well within the quasiparticle roll-off. These are the NEP values shown in Fig. 1c. The measurements shown are taken at the readout power that gives the minimum NEP for that radiation power.

wave readout signal ${ }^{19,20}$. A fit to the measured data gives $\tau_{\mathrm{qp}} \propto P_{\text {read }}^{-0.2 \pm 0.05}$.

Microwave absorption leads to a strong non-equilibrium quasiparticle distribution in aluminium microwave resonators, which was recently shown in calculations ${ }^{20}$ and experimentally ${ }^{28}$. The complex conductivity is shown to be more sensitive to the details of the non-equilibrium quasiparticle distribution than $\tau_{\mathrm{qp}}$. Therefore, the different exponents for $d A / d P_{\text {rad }}$ and $\tau_{\mathrm{qp}}$ in Fig. 5 are not surprising. An extension of the work in ref. 20, including pair-breaking radiation, would be needed to understand the data in Fig. 5. If we make two assumptions, we can get a rough estimate of how the observables in Fig. 5 scale with $P_{\text {read. }}$ We assume that the absorbed microwave power is proportional to $P_{\text {read }}$ and that $\eta_{\text {read }}$, the efficiency with which the readout power is converted to quasiparticles, is constant. In that case, the optical responsivity is expected to scale with readout power as $d A / P_{\text {rad }} \propto P_{\text {read }}^{-0.5}$ in the regime where the readout power dominates the number of quasiparticles, and $\tau_{\mathrm{qp}} \propto P_{\text {read }}^{-0.5}$ (see Supplementary Note 3 for a derivation). Although the scaling of $d A / d P_{\text {rad }}$ is in reasonable agreement with the measurements, the approximation does not cover the different exponents for responsivity and $\tau_{\mathrm{qp}}$. A more detailed theoretical treatment of this problem is required.

\section{Discussion}

At higher radiation powers, where the noise spectrum is dominated by photon noise, the optical responsivity also changes with readout power. In this regime $\left(P_{\mathrm{rad}}>1 \mathrm{fW}\right)$ however, the measured photon noise NEP stays the same, as expected from equation (4) (Supplementary Fig. S4). Therefore, when photon noise dominates the noise spectrum, one can safely use high readout powers to suppress amplifier noise (Supplementary Note 4).

At the lowest readout power where $\tau_{\mathrm{qp}}$ was determined, $P_{\text {read }}=-102 \mathrm{dBm}$, the quasiparticle recombination time is $3.5 \mathrm{~ms}$,

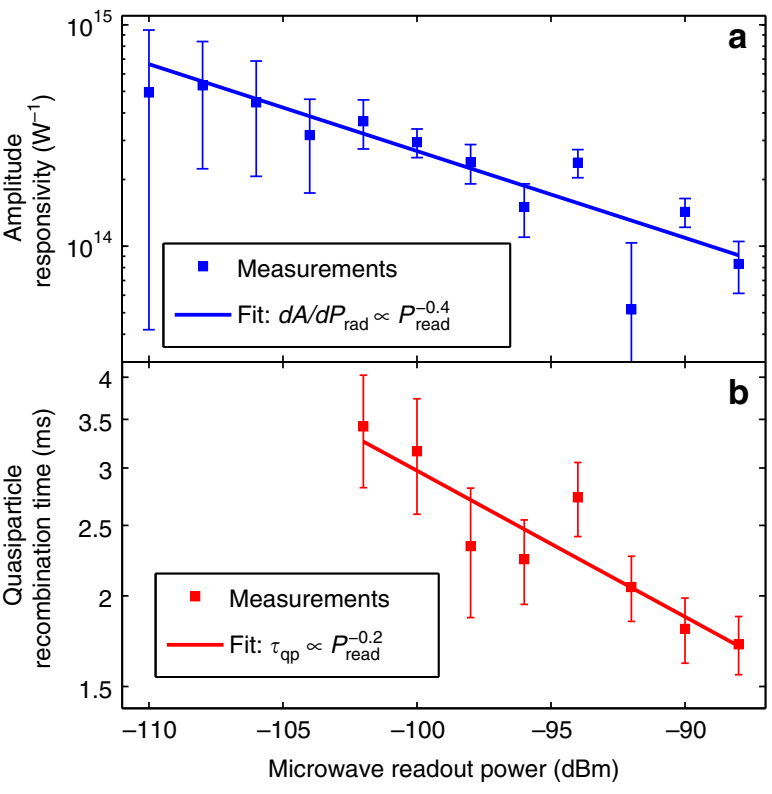

Figure 5 | Readout power dependence. (a) The optical responsivity, as obtained from a fit to the measured amplitude response, as a function of microwave readout power. The error bars denote statistical uncertainties from the fitting procedure $(1$ s.d.). The line is a power law fit to the data: $d A / d P_{\text {rad }} \propto P_{\text {read }}^{-0.4 \pm 0.1}$. (b) The quasiparticle recombination time as obtained from the roll-off in the noise spectra as a function of microwave readout power. The error bars are $1 \mathrm{s.d}$. Below $-102 \mathrm{dBm}$, the roll-off due to the recombination time is not visible anymore. The line is a fit to the data with a power law coefficient of $\tau_{\mathrm{qp}} \propto P_{\text {read }}^{-0.2 \pm 0.1}$. All data are measured at a radiation power of $3 \mathrm{zW}$ and a bath temperature of $120 \mathrm{mK}$.

which corresponds to a quasiparticle density $n_{\mathrm{qp}}=24 \mu \mathrm{m}^{-3}$. This density is still high in comparison with the lowest reported values for superconducting qubits and Cooper-pair transistors ${ }^{17,18}$ (less than $0.1 \mu \mathrm{m}^{-3}$ ), but inherent to the relatively high microwave powers we need in this type of experiments. The measured limit in optical NEP due to excess quasiparticles is comparable with the lowest observed optical NEP in other detectors for similar wavelengths ${ }^{29-31}$.

A reduction in $N_{\mathrm{qp}}$ is possible by using a parametric amplifier with high bandwidth and dynamic range ${ }^{32}$. This allows a reduction of the readout power by about a factor 10 . In the current design, however, the detector would become too slow for practical use at low readout power due to the long recombination time. The most feasible route towards lower NEP with aluminium, the most reliable material so far, is to choose geometries in which the active volume is dramatically reduced, which could also be the route towards single photon counting at terahertz frequencies.

\section{Methods}

Sample design. A layer of aluminium with a thickness of $100 \mathrm{~nm}$ is sputtered onto a sapphire substrate and serves as the ground plane for the microwave resonators. The microwave resonator is a coplanar waveguide resonator with a central strip width of $3 \mu \mathrm{m}$ and slit widths of $1.5 \mu \mathrm{m}$. The central strip of the resonator is made of a second layer of $50 \mathrm{~nm}$ thick Al. The critical temperature of the $50 \mathrm{~nm}$ layer is measured to be $T_{\mathrm{c}}=1.24 \mathrm{~K}$, from which the energy gap $\Delta=1.76 k_{\mathrm{B}} T_{\mathrm{c}}=188 \mu \mathrm{eV}$. From the normal state resistivity, $(\rho=2.2 \mu \Omega \mathrm{cm}$ for the central strip and $0.28 \mu \Omega \mathrm{cm}$ for the ground plane) the skin depth for radiation at $1.54 \mathrm{THz}$ is $60 \mathrm{~nm}$ in the central strip and $21 \mathrm{~nm}$ in the ground plane. The X-slot antenna would be ineffective for a layer thinner than the skin depth, therefore the ground plane layer is $100 \mathrm{~nm}$ thick. The microwave sheet resistance of the central line is $0.46 \Omega$ and that of the ground plane $0.13 \Omega$. Taking this effect into account, together with the participation ratios of the central line and the ground plane ${ }^{33}$, we estimate that $90 \%$ of the radiation is absorbed in the central line. 
The current distribution along the length of the resonator peaks at the antenna and decreases as $\sin (x)$ to zero at the open ends. Therefore, the responsivity changes with $\sin ^{2}(x)$. Since the diffusion length within a typical quasiparticle recombination lifetime of $2 \mathrm{~ms}$ is more than half the resonator length, optically created quasiparticles can move into the non-responsive regime. Therefore, for calculating the number of quasiparticles in the sensitive volume, we take half the central strip volume, $V=0.6 \times 10^{3} \mu \mathrm{m}^{3}$.

Noise measurement. The signal from the microwave generator is first attenuated, sent through the sample and amplified with a HEMT amplifier at $4 \mathrm{~K}$ and with a room temperature amplifier. The output is mixed with the original signal using an IQ mixer, the output of which can be sampled at a maximum frequency of $2 \mathrm{MHz}$. The spectrum of fluctuations in the resonator amplitude is measured by recording the resonator amplitude as a function of time and computing the power spectral density. Peaks in the time domain stream that occur due to high energy impacts are filtered out before the spectrum is computed, as described in ref. 11. We use the amplitude direction because fluctuations in the phase direction are dominated by two level system noise in the dielectrics surrounding the resonator ${ }^{6}$ (Supplementary Fig. S5 and Supplementary Note 1).

Optical system and radiation power. The photon source used in the experiment is a blackbody that is formed by a $40 \mathrm{~mm}$ diameter copper cone, coated with carbon-loaded epoxy (EPOTEK 920 1LB part A, with 3\% by weight carbon black and 3\% by weight EPOTEK $9201 \mathrm{LB}$ part B) that is covered with $1 \mathrm{~mm} \mathrm{SiC} \mathrm{grains.}$ The temperature of the blackbody is varied in this experiment from $3-25 \mathrm{~K}$. There are three metal-mesh filter stacks (QMC Instruments, Cardiff), the characteristics of which are given in Fig. 2a. The measured transmission of the whole filterstack as a function of optical frequency, $\operatorname{Tr}(F)$, is also shown in Fig. 2a.

Since there is no aperture limitation in between the blackbody and the detector, the optical throughput is assumed to be $(c / F)^{2}$, with $c$ the speed of light. The total radiation power that arrives in front of the lens of the detector can now be calculated by numerically integrating Planck's law over the throughput and the measured filter characteristic at each blackbody temperature $T_{\mathrm{BB}}$. The radiation power is here given for one polarization.

$$
P_{\mathrm{rad}}\left(T_{\mathrm{BB}}\right)=\int_{0}^{\infty} \frac{\operatorname{Tr}(F) h F d F}{\exp \left(h F / k_{\mathrm{B}} T_{\mathrm{BB}}\right)-1} .
$$

The optical window around $1.54 \mathrm{THz}$, together with the blackbody temperature range of $3-25 \mathrm{~K}$ gives a large tuning range in radiation power, as shown in Supplementary Fig. S1b. With the present device, we can verify the radiation power down to $0.1 \mathrm{fW}$ using the measured quasiparticle recombination time (Fig. 5b). The trend of increasing optical responsivity and recombination time with decreasing microwave power, without any sign of saturation, suggests that the optical system is well characterized down to even lower radiation powers. The excess quasiparticles in the present device limit us to verify that.

Right in front of the detector, after the last optical filter, we place a polarizer to select the polarization for which the antenna is designed. The polarizer consists of a copper wire grid on top of a $1.5-\mu \mathrm{m}$ thick Mylar film. The grid lines are $10 \mu \mathrm{m}$ wide and the spacing between the lines is $20 \mu \mathrm{m}$.

Responsivity fit. The responsivity $d A / d P_{\text {rad }}$ is obtained from a linear fit to the response $A$ measured as a function of $P_{\mathrm{rad}}$. The blackbody temperature is swept slowly around the $P_{\text {rad }}$ of interest. Each measured point is integrated for $500 \mathrm{~ms}$. At very low radiation power $\left(P_{\text {rad }}<0.3 \mathrm{aW}\right)$ there is no response (due to the excess quasiparticles). Therefore, the responsivity at $3 \mathrm{zW}$ is estimated by a fit of the response in the range 0.3-9 aW, for example. Note that this reflects the saturation in the sensitivity of the detector. At the highest power $P_{\mathrm{rad}}=724 \mathrm{fW}$, the fit range is 705-743 fW. The fit range is chosen by hand, because it differs widely from the saturation regime to the regime in which the number of quasiparticles is dominated by the absorbed $P_{\text {rad }}$. The fit range is chosen small enough to stay in the linear response regime and large enough to limit the standard deviation of the fit.

Lens antenna efficiency. The radiation power is focused by an elliptical silicon lens of $2 \mathrm{~mm}$ in diameter onto the antenna that is in the second focus of the lens ${ }^{34}$. The major and minor axes of the ellipse are 1.037 and $0.992 \mathrm{~mm}$, respectively. The lens has an antireflection coating of $130 \mu \mathrm{m}$ of Parylene C, which is not optimized for $1.54 \mathrm{THz}$. The antenna is an in-line X-slot antenna, designed to receive radiation in a broad band around $1.54 \mathrm{THz}$ as described in ref. 21. To obtain the optical efficiency, a simulation in CST Microwave Studio is performed of the whole structure: the lens with the coating, the antenna and a piece of coplanar waveguide transmission line. The aperture is chosen to be 30 degrees, the angle from which the detector can see the blackbody (single-side angle). The optical efficiency is shown as a function of frequency in Supplementary Fig. S6. The total efficiency is the multiplication of the front-to-back ratio, the spill over losses, the efficiency of an impedance mismatch between the antenna and the CPW line and the reflection losses at the anti-reflection-coated lens surface. All together, we expect an efficiency of $48 \%$ for one polarization in the filter transmission band, which is in good agreement with the measured optical efficiency of $48 \pm 8 \%$. The antenna was not designed to have a perfect optical efficiency, but to have a large bandwidth. The agreement of the measured optical efficiency with the CST-simulation shows that the optical system is understood.

\section{References}

1. Day, P. K., LeDuc, H. G., Mazin, B. A., Vayonakis, A. \& Zmuidzinas, J. A broadband superconducting detector suitable for use in large arrays. Nature 425, 817-821 (2003).

2. Mattis, D. C. \& Bardeen, J. Theory of the anomalous skin effect in normal and superconducting metals. Phys. Rev. 111, 412-417 (1958).

3. Kaplan, S. B. et al. Quasiparticle and phonon lifetimes in superconductors. Phys. Rev. B 14, 4854-4873 (1976)

4. Yates, S. J. C. et al. Photon noise limited radiation detection with lens-antenna coupled microwave kinetic inductance detectors. Appl. Phys. Lett. 99, 073505 (2011).

5. Zmuidzinas, J. Superconducting microresonators: physics and applications. Ann. Rev. Condens. Matter Phys. 3, 169-214 (2012).

6. Gao, J., Zmuidzinas, J., Mazin, B. A., LeDuc, H. G. \& Day, P. K. Noise properties of superconducting coplanar waveguide microwave resonators. Appl. Phys. Lett. 90, 102507 (2007).

7. Gao, J. et al. A semiempirical model for two-level system noise in superconducting microresonators. Appl. Phys. Lett. 92, 212504 (2008).

8. Barends, R. et al. Contribution of dielectrics to frequency and noise of NbTiN superconducting resonators. Appl. Phys. Lett. 92, 223502 (2008).

9. Barends, R. et al. Minimal resonator loss for circuit quantum electrodynamics. Appl. Phys. Lett. 97, 033507 (2010).

10. Wilson, C. M., Frunzio, L. \& Prober, D. E. Time-resolved measurements of thermodynamic fluctuations of the particle number in a nondegenerate Fermi gas. Phys. Rev. Lett. 87, 067004 (2001).

11. de Visser, P. J. et al. Number fluctuations of sparse quasiparticles in a superconductor. Phys. Rev. Lett. 106, 167004 (2011).

12. Martinis, J. M., Ansmann, M. \& Aumentado, J. Energy decay in superconducting Josephson-junction qubits from nonequilibrium quasiparticle excitations. Phys. Rev. Lett. 103, 097002 (2009).

13. Catelani, G. et al. Quasiparticle relaxation of superconducting qubits in the presence of flux. Phys. Rev. Lett. 106, 077002 (2011).

14. Lenander, M. et al. Measurement of energy decay in superconducting qubits from nonequilibrium quasiparticles. Phys. Rev. B 84, 024501 (2011).

15. Zgirski, M. et al. Evidence of long-lived quasiparticles trapped in superconducting point contacts. Phys. Rev. Lett. 106, 257003 (2011).

16. Barends, R. et al. Minimizing quasiparticle generation from stray infrared light in superconducting quantum circuits. Appl. Phys. Lett. 99, 113507 (2011).

17. Saira, O.-P., Kemppinen, A., Maisi, V. F. \& Pekola, J. P. Vanishing quasiparticle density in a hybrid Al/Cu/Al single-electron transistor. Phys. Rev. B 85, 012504 (2012).

18. Ristè, D. et al. Millisecond charge-parity fluctuations and induced decoherence in a superconducting qubit. Nat. Commun. 4, 1913 (2013).

19. de Visser, P. J. et al. Microwave-induced excess quasiparticles in superconducting resonators measured through correlated conductivity fluctuations. Appl. Phys. Lett. 100, 162601 (2012).

20. Goldie, D. J. \& Withington, S. Non-equilibrium superconductivity in quantum-sensing superconducting resonators. Supercond. Sci. Technol. 26, 015004 (2013).

21. Iacono, A., Freni, A., Neto, A. \& Gerini, G. In-line X-slot element focal plane array of kinetic inductance detectors. In: Proceedings of the European Conference on Antennas and Propagation (Rome, Italy, 2011).

22. Baselmans, J., Yates, S., Diener, P. \& de Visser, P. Ultra low background cryogenic test facility for far-infrared radiation detectors. J. Low Temp. Phys. 167, 360-366 (2012)

23. Boyd, R. W. Photon bunching and the photon-noise-limited performance of infrared detectors. Infrared Phys. 22, 157-162 (1982).

24. Wilson, C. M. \& Prober, D. E. Quasiparticle number fluctuations in superconductors. Phys. Rev. B 69, 094524 (2004).

25. Kozorezov, A. G. et al. Quasiparticle-phonon downconversion in nonequilibrium superconductors. Phys. Rev. B 61, 11807 (2000).

26. Gao, J. et al. Equivalence of the effects on the complex conductivity of superconductor due to temperature change and external pair breaking. J. Low Temp. Phys. 151, 557-563 (2008).

27. Barends, R. et al. Enhancement of quasiparticle recombination in Ta and $\mathrm{Al}$ superconductors by implantation of magnetic and nonmagnetic atoms. Phys. Rev. B 79, 020509(R) (2009).

28. de Visser, P. J. et al. Evidence of a nonequilibrium distribution of quasiparticles in the microwave response of a superconducting aluminum resonator. Phys. Rev. Lett. (in the press); preprint at http://arXiv.org/abs/1306.4992 (2013).

29. Karasik, B. S. \& Cantor, R. Demonstration of high optical sensitivity in far-infrared hot-electron bolometer. Appl. Phys. Lett. 98, 193503 (2011)

30. Audley, M. D. et al. Measurements of the optical performance of bolometers for SPICA/SAFARI. Proc. SPIE 8452, 84520B-1-84520B-12 (2012). 
31. Stone, K. J. et al. Real time quasiparticle tunneling measurements on an illuminated quantum capacitance detector. Appl. Phys. Lett. 100, 263509 (2012).

32. Eom, B. H., Day, P. K., LeDuc, H. G. \& Zmuidzinas, J. A wideband, low-noise superconducting amplifier with high dynamic range. Nat. Phys. 8, 623-627 (2012).

33. Holloway, C. L. \& Kuester, E. F. A quasi-closed form expression for the conductor loss of CPW lines, with an investigation of edge shape effects. IEEE Trans. Microw Theory Tech. 43, 2695-2701 (1995).

34. Filipovic, D., Gearhart, S. \& Rebeiz, G. Double-slot antennas on extended hemispherical and elliptical silicon dielectric lenses. IEEE Trans Microw. Theory Tech. 41, 1738-1749 (1993).

\section{Acknowledgements}

We would like to thank Y.J.Y. Lankwarden for fabricating the devices and S.J.C. Yates for contributing to the experimental setup. This work has been supported as part of a collaborative project, SPACEKIDS, funded via grant 313320 provided by the European Commission under Theme SPA.2012.2.2-01 of Framework Programme 7.

\section{Author contributions}

J.J.A.B. conceived the experiment. P.J.V., J.J.A.B. and J.B. performed the experiments. N.L. analysed and simulated the optical system. P.J.V., J.J.A.B. and T.M.K. analysed the data and composed the article.

\section{Additional information}

Supplementary Information accompanies this paper at http://www.nature.com/ naturecommunications

Competing financial interests: The authors declare no competing financial interests.

Reprints and permission information is available online at http://www.npg.nature.com/ reprintsandpermissions/

How to cite this article: de Visser, P. J. et al. Fluctuations in the electron system of a superconductor exposed to a photon flux. Nat. Commun. 5:3130 doi: 10.1038/ ncomms4130 (2014).

(c) (1) $\ominus$ This work is licensed under a Creative Commons Attribution(cc) $\$$ BY NC ND NonCommercial-NoDerivs 3.0 Unported License. To view a copy of this license, visit http://creativecommons.org/licenses/by-nc-nd/3.0/ 